\title{
Mass and Energy Supply of Fine Structure to the Solar Corona
}

\author{
Kostas Tziotziou \\ Sterrekunding Instituut Utrecht, Postbus 80000, 3508 TA Utrecht, The \\ Netherlands \\ Georgia Tsiropoula \\ National Observatory of Athens, Institute for Space Applications and \\ Remote Sensing, Lofos Koufos, 15236 Palea Penteli, Greece
}

\begin{abstract}
We investigate the role of chromospheric fine structures, e.g. mottles (spicules), in the mass balance and heating of the solar atmosphere by studying two-dimensional high-resolution $\mathrm{H} \alpha$ observations. The temporal and spatial variations of the line-of-sight (LOS) velocity, obtained with an inversion technique based on a cloud model, provide strong indications that the mechanism responsible for the driving of the observed flows is magnetic reconnection. Apart from the LOS velocity, application of the cloud model enables the derivation of several other physical parameters, like pressure, temperature, density etc. Mean values of these parameters permit the estimation of the role of these structures in the mass balance of the solar atmosphere. They, furthermore, permit a reasonable estimate of the energy provided by magnetic reconnection which is available for the heating of the solar corona.
\end{abstract}

\section{Introduction}

Mottles are rapidly changing hairlike jets of relatively cool material ejected from the low chromosphere into the hot corona with supersonic velocities of the order of $20-30 \mathrm{~km} \mathrm{~s}^{-1}$. Their properties are similar to those of spicules seen at the limb (Tsiropoula \& Schmieder 1997, hereafter Paper I) and thus we shall use the terms mottles and spicules indifferently. A comprehensive review of mottle properties can be found in Beckers (1972).

Mottles usually cluster into small groups which are called chains and larger groups which are called rosettes that outline the boundaries of the chromospheric network where strong mixed polarity magnetic flux is mostly concentrated. It is well established that bipolar elements emerge continuously inside the network cells and are swept to the network boundaries by the supergranular flow (Wang et al. 1996; Schrijver et al. 1997), where interactions of the magnetic fields have as a result the enhancement of the flux concentration in the case of same polarities or its cancellation in the case of opposite polarities. The latter most likely involves magnetic reconnection which would drive material out of the reconnection region in opposite directions. 

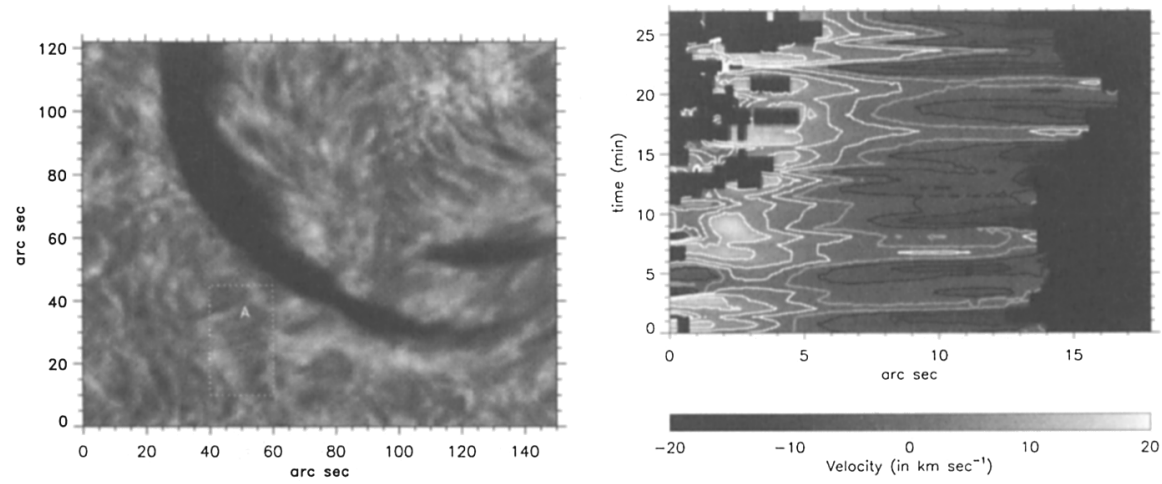

Figure 1. Left: An intensity image at $\mathrm{H} \alpha+0.24 \AA$ of the observed area. The dotted rectangular area indicates the mottles used for this study. Right: Time slice image of the LOS velocity $v$ for a cut along the central axis of the mottle marked as A on the left image. Black contours denote upward velocities (negative), white contours downward velocities while the thick gray line represents the zero velocity contour. Position " 0 "" corresponds to the foot of the structure.

Recently using THEMIS/MSDP spectrograph data (Tziotziou, Tsiropoula, \& Mein 2003, hereafter Paper II) in conjunction with a cloud model, we derived physical parameters of the dark mottles and their temporal evolution. From the temporal evolution of the LOS velocity we found that the predominant motion is downward at their footpoints and alternatively upward and downward at their tops. Based on these observational findings we proposed a phenomenological model according to which mottles are due to magnetic reconnection. We also found a recurrent behaviour for intensity and velocity variations with a period of $\sim 5$ min.

Magnetic reconnection is probably the most suitable mechanism not only for releasing energy into the corona, but also for the transfer of cool gas from the chromosphere to the corona and the solar wind. Withbroe \& Noyes (1977) have estimated that energy fluxes of $510^{6} \mathrm{ergs} \mathrm{cm}^{-2} \mathrm{~s}^{-1}$ and $310^{5} \mathrm{ergs} \mathrm{cm}^{-2} \mathrm{~s}^{-1}$ are needed to maintain the quiet chromosphere and the quiet corona respectively, while the typical mass flux estimate needed to maintain the solar wind is $310^{-11} \mathrm{gr} \mathrm{cm}^{-2} \mathrm{~s}^{-1}$ (Ulmschneider 1971). The aim of this work is to obtain estimates of the role of mottles in the mass balance and energy budget of the solar atmosphere.

\section{Observations and analysis procedure}

A time series of a $150^{\prime \prime} \times 120^{\prime \prime}$ solar region (see Fig. 1, left image) containing 40 frames with a cadence of $\sim 40.5 \mathrm{~s}$ was obtained in $\mathrm{H} \alpha$ on May 14th, 2000 with the THEMIS/MSDP spectrograph. For each of our two-dimensional images we have the line intensity at line center, $\pm 0.24 \AA$ and $\pm 0.48 \AA$ and Doppler shifts at $\pm 0.24 \AA$ and $\pm 0.48 \AA$. 
Among the most widely applied methods for the deduction of different physical parameters of optically thin chromospheric structures is the cloud model technique (Beckers 1964), which is based on the comparison of $\mathrm{H} \alpha$ line profiles emitted by the body of the structures with those emitted below the structures. Using an iterative least-square procedure for non-linear functions (see Paper II) we can deduce the values of the source function $S$, the Doppler width $\Delta \lambda_{\mathrm{D}}$, the optical thickness $\tau_{0}$ and the velocity $v$ that best describe an observed profile. Once these four parameters are determined several other parameters can be estimated, like the temperature $T$, the number density of the second hydrogen level $N_{2}$, the electron density $N_{\mathrm{e}}$, the total particle density of hydrogen (i.e., neutral plus ionized) $N_{\mathrm{H}}$, the ionization degree $\chi$, the gas pressure $p$, the total column mass $M$ and the mass density $\rho$, assuming a value for the microturbulent velocity $\xi_{\mathrm{t}}$ and using the relations described in Paper I.

\section{Results}

The mean values of some physical parameters of the dark mottles that appear in the dotted rectangular area shown in Fig. 1 for the entire sequence are: $N_{2}$ $=4.210^{4} \mathrm{~cm}^{-3}, N_{\mathrm{e}}=6.410^{10} \mathrm{~cm}^{-3}, \rho=2.210^{-13} \mathrm{~g} \mathrm{~cm}^{-3}, \chi=0.65, M=$ $2.210^{-5} \mathrm{~g} \mathrm{~cm}^{-2}, p=0.24{\mathrm{dyn} \mathrm{cm}^{-2}}^{2}$ and $T=10^{4} \mathrm{~K}$. For the estimation of the temperature a microturbulent velocity, $\xi_{\mathrm{t}}$, equal to $15 \mathrm{~km} \mathrm{~s}^{-1}$ was assumed. The mean values of the length $L$, width $d$, and inclination angle $\theta$, of the mottles have been taken to be equal to $10 \mathrm{Mm}, 1 \mathrm{Mm}$ and $45^{\circ}$ respectively.

In order to examine the time evolution of the physical parameters along individual mottles, their values were averaged over a strip extending $0.3^{\prime \prime}$ on either side of the central axes of several mottles which were carefully traced on each image. An example of the temporal evolution of the cloud velocity (time slice image) along the axis of a distinct mottle is given in Fig. 1 (right). It shows that mottles have a bi-directional flow structure showing predominantly downward motion at their footpoints and upward at their tops. This behaviour has been explained in Paper II as the result of magnetic reconnection.

For the evaluation of the role of mottles in the mass and energy balance of the solar atmosphere, we will take the mean values of the parameters given above as representative ones. We also remind that mottles arise at the network boundaries as bursts of material attaining maximum upward or downward LOS velocities $v$ of the order of $15 \mathrm{~km} \mathrm{~s}^{-1}$ and tend to occur several times at the same location with a typical duration of $\sim 5 \mathrm{~min}$.

\subsection{The role of mottles in the mass balance of the solar atmosphere}

The upward mass flux of mottles depends on: a) the fraction, $f$, of the solar disk covered by these structures at any given moment, b) the mass density, $\rho$, and c) the axial upward mottle velocity, $v_{\alpha}$. By counting the mottles in the observed $150^{\prime \prime} \times 120^{\prime \prime}$ area we have estimated the total number of mottles on the solar surface to be equal to $510^{4}$ and the fraction of the solar surface they cover to $\sim 0.024$. The axial velocity $v_{\alpha}$ is $21 \mathrm{~km} \mathrm{~s}^{-1}\left(v_{\alpha}=v / \cos \theta\right)$. Assuming that at any given moment only half of the mottles are showing upward motion and from them only half of the material is flowing upwards, the globally averaged 
mass flux carried upwards obtained from the relation

$$
F_{M}=\frac{1}{2}\left(\frac{1}{2} f \rho v_{\alpha}\right)
$$

is equal to $2.810^{-9} \mathrm{~g} \mathrm{~cm}^{-2} \mathrm{~s}^{-1}$. This value is two orders of magnitude larger than the outward flow of mass from the corona due to the solar wind which is equal to $310^{-11} \mathrm{~g} \mathrm{~cm}^{-2} \mathrm{~s}^{-1}$ (Ulmschneider, 1971). Thus it seems that these structures provide the means by which coronal mass losses in the solar wind can be compensated by mass ejected from below establishing the overall mass balance in the solar atmosphere. We must also conclude that only $1 \%$ of the material of mottles escapes. The remainder returns back to the chromosphere which could explain the downflows observed in EUV lines (Pneumann \& Kopp 1978; Withbroe 1983).

\subsection{The role of mottles in the energy budget of the solar corona}

We consider that the released energy is furnished by magnetic dissipation. The most effective and plausible mechanism for this dissipation is magnetic reconnection. We assume that the energy produced by magnetic dissipation at the reconnection site goes into heating, kinetic energy of the bulk flow, gravitational energy, as well as conduction and radiation losses. We also consider that the part of the structure below the reconnection point is pulled down and thus the energy provided by the dissipation of the magnetic energy goes to the heating of the chromosphere, while the energy dissipated above the reconnection point goes to the heating of the corona. Thus we separate the energy fluxes into those travelling away and those travelling towards the observer according to the upward $v_{-}$and downward $v_{+}$velocity directions. We, furthermore, consider two phases in the lifetime of a mottle: a) the reconnection phase and b) the downflow phase. We, finally, consider the energy balance per event and then integrate over the solar surface.

During the reconnection phase the energy balance per event requires that

$$
\begin{aligned}
F_{\mathrm{m} \pm} & =F_{\mathrm{rd} \pm}+F_{\text {cond } \pm}+F_{\mathrm{kin} \pm}+F_{\mathrm{h} \pm}+F_{\mathrm{g} \pm} \\
& =e_{\mathrm{rd}} \frac{L}{2}+\kappa \frac{\mathrm{d} T}{\mathrm{~d} h}+\frac{1}{2} \rho v_{ \pm}^{3}+\frac{\gamma p}{(\gamma-1)} v_{ \pm} \mp \rho g \frac{L \cos (\theta)}{2} v_{ \pm}
\end{aligned}
$$

expressed in terms of fluxes, e.g. in erg $\mathrm{cm}^{-2} \mathrm{~s}^{-1}$, (energy per unit surface per unit time). The first term of Eq. 2 corresponds to the radiative flux. For the net radiative loss rate per unit volume $e_{\mathrm{r}}$ we use the analytical expression given by Nagai (1980), $e_{r} d=\alpha(T) N_{\mathrm{e}}^{2} \chi g(T)$ where $g(T)$ is a semiempirical function of the temperature and $\alpha$ is the radiative coefficient. For $\mathrm{T}=$ $10^{4} \mathrm{~K}, g(T) \sim 10^{-23} \mathrm{erg} \mathrm{cm}^{3} \mathrm{~s}^{-1}$ and $\alpha(T) \sim 0.1$, the radiative flux is equal to $1.2610^{6} \mathrm{erg} \mathrm{cm}^{-2} \mathrm{~s}^{-1}$. The second term of Eq. 2 corresponds to the conductive flux along magnetic field lines where $\kappa=1.110^{-6} \mathrm{~T}^{5 / 2} \mathrm{erg} \mathrm{s}^{-1} \mathrm{~cm}^{-1} \mathrm{~K}^{-7 / 2}$ is the Spitzer thermal conductivity. Equating the relation above to $1.110^{-6} \mathrm{~T}^{7 / 2} /\left(\frac{L}{2}\right)$, a conductive flux equal to $0.22 \mathrm{ergs} \mathrm{cm}^{-2} \mathrm{~s}^{-1}$ is obtained. It should be noted that both radiative and conductive fluxes are upper limits since we assume that the entire volume radiates or conducts the energy. The third term of Eq. 2 represents the kinetic energy flux, the fourth the enthalpy flux and the fifth the 
gravitational energy flux with $\gamma=\frac{5}{3}$ being the ratio of the specific heats and $g$ the acceleration due to solar gravity. We obtain that the kinetic energy flux per event is equal to $1.0210^{6} \mathrm{ergs} \mathrm{cm}^{-2} \mathrm{~s}^{-1}$, the enthalpy flux per event is of the order of $1.2610^{6} \mathrm{ergs} \mathrm{cm}^{-2} \mathrm{~s}^{-1}$ and the gravitational energy flux per event is equal to $6.3210^{6} \mathrm{ergs} \mathrm{cm}^{-2} \mathrm{~s}^{-1}$.

Adopting for the duration of the reconnection phase of each event a time $t_{\mathrm{i}}$ equal to $150 \mathrm{~s}$, the contribution of all mottles to the global energy flux can be found from the total upward or downward individual energy fluxes by the relation

$$
F_{\mathrm{t}, \text { glob l, } \pm}=\frac{F_{\mathrm{t}, \mathrm{i} \pm} S_{\mathrm{i}} t_{\mathrm{i}} b}{2 S_{\mathrm{o}}}
$$

where $S_{\mathrm{i}}$ is the area of the event, $b$ the birthrate of mottles - which is obtained from their total number seen on the disk, divided by their mean lifetime $(b=167$ events/sec) - and $S_{\mathrm{o}}$ the surface of the Sun. The total energy obtained by this relation is equal to $2.510^{5} \mathrm{ergs}^{-2}$. On the other hand, the energy outflow available for heating the solar corona, that is the total kinetic and enthalpy flux, is of the order of $3.610^{4} \mathrm{ergs} \mathrm{cm}-2$.

\section{Conclusion}

The role of fine structures is an essential ingredient for the modeling of the solar atmosphere. Our estimates show that the mass outflow from chromospheric mottles is $\sim 2$ orders of magnitude larger than that in the solar wind and hence coronal mass losses can be compensated by mass supply from such structures. On the other hand evaluating the global energetics associated with mottles we estimated that the flux available for heating the solar corona is only $\sim 12 \%$ of the required energy. This means that the corona could in fact be heated by a variety of mechanisms.

\section{References}

Beckers, J.M. 1964, A study of the fine structures in the solar chromosphere, Ph.D. Thesis, Utrecht

Beckers, J.M. 1972, ARA\&A, 10, 73

Nagai, F. 1980, Solar Phys. 68, 351

Pneumann, G.W., \& Kopp, R.A. 1978, Solar Phys., 57, 49

Schrijver, C.J., Title, A.M., Van Ballegooijen, A.A., Hagenaar, H.J., \& Shine, R.A. 1997, ApJ, 487, 424

Tsiropoula, G., \& Schmieder, B. 1997, A\&A, 324, 1183 (Paper I)

Tziotziou, K., Tsiropoula, G., \& Mein, P. 2003, A\&A, 402, 361 (Paper II)

Ulmschneider, P. 1971, A\&A, 12, 297

Wang, H., Tang, F., Zirin, H., \& Wang, J. 1996, Solar Phys., 165, 223

Withbroe, G.L. 1983, ApJ, 267, 825

Withbroe, G. L., \& Noyes, R. W. 1977, ARA\&A, 15, 363 